Supplemento n. 3

Giornale di Tecniche

Nefrologiche \& Dialitiche

Anno XIV n. 2

(C) Wichtig Editore 2002

\title{
Il tallone d'Achille della moderna emodialisi
}

\section{Marco Lombardi}

\section{U.O. di Nefrologia e Dialisi, Ospedale S.M. Annunziata, Azienda USL, Firenze}

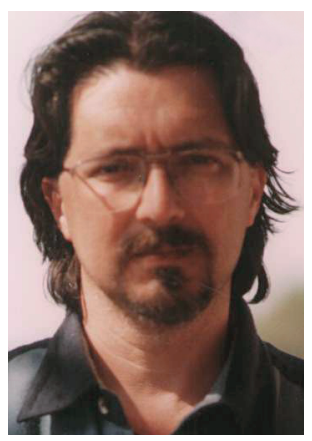

OGGI

La rapida crescita dei programmi di trattamento dell'uremia terminale in tutto il mondo ha reso ancor più evidente, con il crescente aumento della morbilità degli accessi vascolari per emodialisi (2), quello che è definito da tempo "the Achilles heel of the modern hemodialysis" (3). I pazienti uremici incidenti presentano sempre più spesso severe condizioni di comorbidità (diabete (Fig. 1), malattie vascolari periferiche ecc.) che si ripercuotono aspramente sulla "fallacità" degli accessi vascolari per dialisi; per non parlare dell'influenza del cambiamento demografico in atto da tempo (Fig. 1) (4, $5)$.

Si tratta di un notevole problema senza apparenti soluzioni a portata di mano, un problema dibattuto tra "addetti ai lavori", medici (nefrologi, chirurghi vascolari, radiologi interventisti ecc.), infermieri e ovviamente pazienti, per la soluzione del quale è auspicato anche l'intervento di ricercatori di altre branche scientifiche, come ingegneri biomedici o quant'altri sia necessario coinvolgere. Infatti, l'inadeguatezza dell'accesso vascolare oltre che porre in campo una serie di problematiche multidisciplinari, produce un riverbero economico che non potrà esser ignorato ancora per molto (6). Basti pensare, che mentre all'inizio degli anni '90, nei pazienti emodializzati le ospedalizzazioni correlate a una patologia dell'accesso vascolare non superavano il 25\% (3), oggi queste arrivano al 50\% (7), con ovvie implicazioni d'allocabilità delle risorse e sociali. Negli USA a esempio, nel 1995, Medicare registrava un costo superiore al miliardo di dollari per gli oltre 90,000 ricoveri correlati a problemi d'accesso vascolare per dialisi (5).

\section{Adeguatezza}

È un termine molto usato, che la dice lunga su quanto siamo, purtroppo, ancora lontani dall'eccellenza nella cura dei pazienti uremici cronici in emodialisi ("un'entità superiore" non $a c$ crediterebbe le nostre attuali soluzioni d'accesso vascolare in dialisi!). Adeguatezza, certo, perché a tutt'oggi non è possibile parlare d'accesso, vascolare ideale in emodialisi, ovvero quel dispositivo che dovrebbe porre in comunicazione l'albero vascolare del paziente, con il circuito extracorporeo della macchina, fornendo un flusso ematico ottimale, costantemente per un tempo indefinito. L'adeguatezza dell'accesso vascolare (naturalmente non solo questa) è infatti in molti casi la principale barrierra al raggiungi-

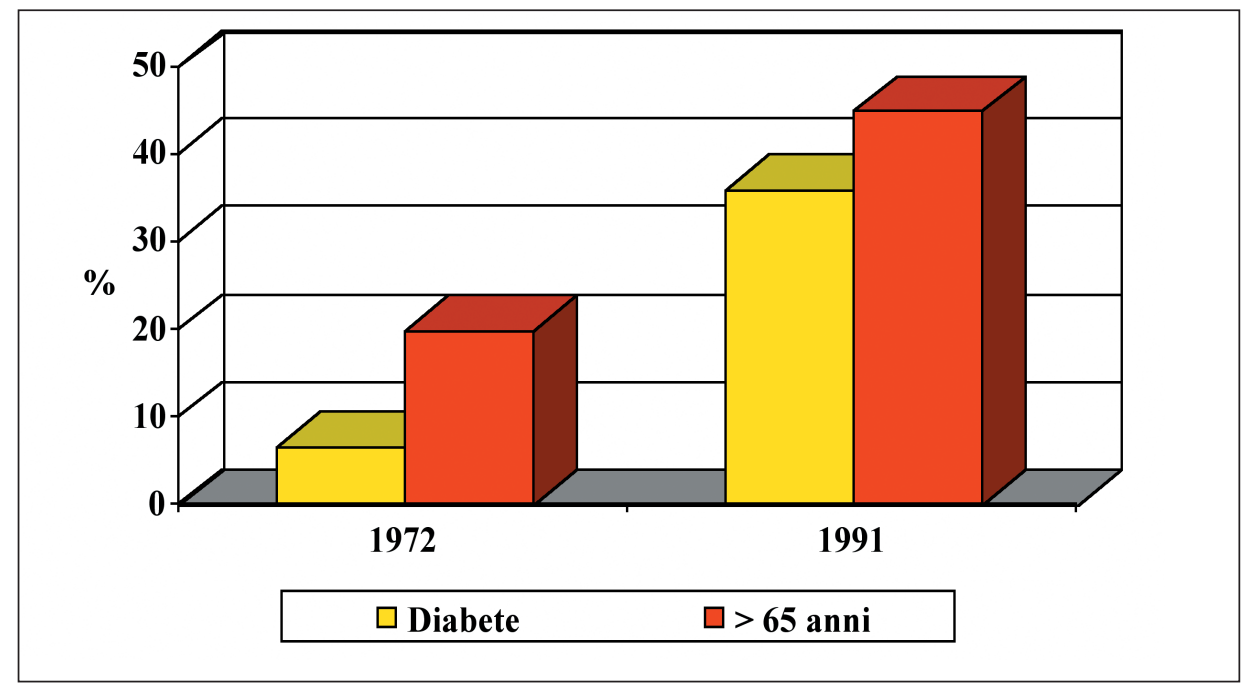

Fig. 1 - Cambiamento di comorbidità (diabete m.) e demografico nella popolazione uremica in dialisi, negli USA (5). 


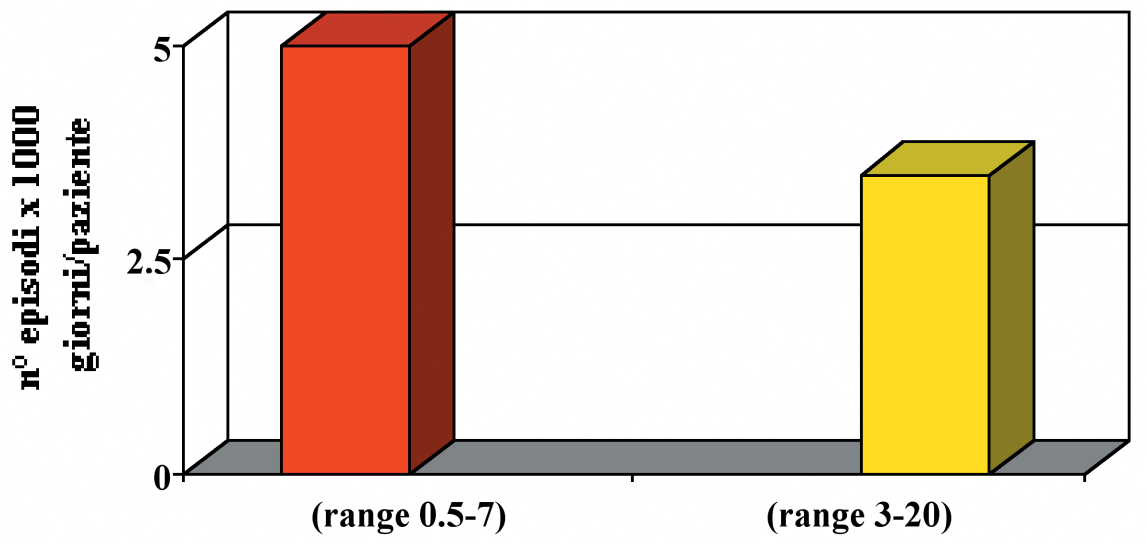

$\square$ CVC temporaneo $\square$ CVC permanente

mento dell'adeguatezza dialitica (7). Una cosa non da poco se si pensa che morbilità, mortalità e quindi ospedalizzazioni (alias aggravio della spesa per la comunità), ma anche sofferenze e condizioni socio-lavorative (alias qualità di vità degli emodializzati) sono strettamente correlate all'adeguatezza dialitica.

\section{IERI}

Se è vero che non basterà risolvere la problematica degli accessi vascolari per sistemare tutto, è pur vero che da decenni non vengono trovate prospettive veramente innovative e rivoluzionarie, come lo fu a suo tempo la fistola A-V autologa di Cimino e Brescia (8), l'ultima, vera, grande "scoperta" nel campo degli accessi vascolari per emodialisi, e attuale gold standard (4, 9).

Fig. 2 - Incidenza media di infezioni correlate a catetere venoso centrale (CVC) per emodialisi (11). Sono poi occorsi quindici anni per l'introduzione della protesi in politetrafluoroetilene (PTFE) (10), che pur essendo di grande aiuto in situazioni particolari, non è certo the final outcome: U.S. docet! Neppure l'introduzione dei cateteri venosi centrali-temporanei e permanenti ha chiuso la partita; a fronte di tante soluzioni raggiunte, sono sorti sempre nuovi e importanti problemi (Fig. 2) (11).

\section{DOMANI}

Sono in atto numerosi ed evidenti sforzi per la progressione nel campo specifico. I più recenti dispositivi tipo Dialock ${ }^{\circledR}$ e e altri similari sono forieri di vantaggi di non poco conto, anche sul piano economico (nonostante il loro attuale costo). Dispositivi del genere saranno in grado di ridurre i tempi e il numero delle ospedalizzazioni, data la facilità d'applicazione e la quasi totale e immediata utilizzabilità (4) ma anche perché la loro diffusione potrebbe permettere l'incremento sia della dialisi domiciliare che soprattutto di quelle forme di dialisi più adeguata (notturna, giornaliera ecc.) (4), che già di per sé sono un'assicurazione di riduzione della morbilità, di oneri socio-economici, di recupero socio-lavorativo e di qualità di vita (12).

$\mathrm{Ma}$ in attesa dell'equivalente della fistola $\mathrm{A}-\mathrm{V}$ del Ventunesimo secolo qualcosa andrà rapidamente migliorato anche sul piano organizzativo.

A esempio, esistono ormai evidenze provate, che per la sua miglior riuscita l'accesso vascolare autologo/eterologo ha bisogno di un certo timely referral; è inoltre risaputo che questo è direttamente correlato al numero di visite nefrologiche che il paziente ha eseguito durante il decorso dell'uremia cronica (13). Poiché questo è un mero problema d'organizzazione e programmazione è sentita dall'autore (ma non solo) la necessità di un movimento d'opinione che partendo dall'organo preposto alla tutela della salute dei cittadini, il Ministero della Salute, passi per le sedi regionali preposte, gli ambulatori di nefrologia, per arrivare ai medici di medicina generale, e il cui motto potrebbe essere: ogni paziente late referral è un'onta comune (oltre che una disgrazia "evitabile", per lo stesso).

Dovrebbe esser la norma, che ogni paziente affetto da insufficienza renale cronica sia tempestivamente riferito in ambito nefrologico, per esser accudito e ottenere tutte le appropriate informazioni del caso tra cui anche quelle per porre in atto il risparmio del proprio patrimonio vascolare: $i$ ) imparando che si possono evitare le venipunture indesiderate $(1,6)$; $i i)$ venendo a conoscenza che si può procrastinare, ove possibile, lo sfruttamento del proprio patrimonio vascolare, grazie all'uso di un trattamento sostitutivo renale diverso dall'emodialisi, ovvero con la dialisi peritoneale (14); iii) potendosi sottoporre in tempo utile all'allestimento dell'accesso per il tipo di dialisi a lui più confacente (1).

\section{Subito}

Una problematica a cascata quella degli accessi vascolari per dialisi; non a caso è stato chiesto a differenti specificità professionali di partecipare alla realizzazione di questo contributo e non a caso hanno risposto molti colleghi, che appartengono e collaborano attivamente con il Gruppo di Studio per gli Accessi Vascolari della Società Italiana di Nefrologia. Alla lettura delle pagine web di questo Gruppo di Studio (15) si avverte quanto sia sentita la necessità di uno strumento in permanente aggiornamento, utile per la valutazione e validazione delle attività più consone allo sviluppo della materia, come la realizzazione di un Registro Nazionale degli Accessi Vascolari. Questo strumento di valutazione e confronto (a 
livello nazionale e sovranazionale) potrebbe esser la base per la creazione di linee guida che siano veramente agganciate a quella realtà che ogni giorno si consuma all'interno delle sale dialisi.

Questi propositi, possenti d'esser nati in seno alla Società scientifica che rappresenta la maggior parte degli addetti ai lavori, dovrebbero essere promossi in maniera forte e rapida, in stretta collaborazione multidisciplinare, coinvolgendo quindi tutte le specificità professionali utili in Sanità (certamente gli infermieri) e non strettamente dell'area sanitaria.

Scopo di questo Supplemento è cercare di alimentare ulteriormente il dibattito scientifico in atto sugli accessi vascolari nella nostra comunità nefrologica, cercando di coinvolgere, con fini volutamente costruttivi tutte le professionalità utili, nella speranza di abbreviare il cammino verso quello che sarà, prima o poi, l'accesso vascolare ideale per il paziente in dialisi.

\section{BIBLIOGRAFIA}

1. Segoloni GP, Mangiarotti G, Pacitti A. La chirurgia degli accessi vascolari. In: Aspetti tecnici in nefrologia. A cura di Andreucci VE, Ac. Naz. Med. 1998, vol.4.

2. May RE, Himmelfarb J, Yenicesu M, et al. Predictive measures of vascular access thrombosis: a prospective study. Kidney Int 1997; 52: 1656-62.

3. Fan PY, Schwab SJ. Vascular access: concepts for the 1990s. J Am Soc Nephrol 1992; 3: 1-11.

4. Quarello F, Forneris G, Formica M, et al. Dialock subcutaneous port for hemodialysis: a twelwe-month experience in a single centre. The Journal of Vascular Access 2001; $2: 73-9$.

5. Feldman HI, Kobrin S, Wasserstein A. Hemodialysis vascular access morbidity. J Am Soc Nephrol 1996; 7: 523-35.

6. Vanholder R. Vascular access: care monitoring of function. Nephrol Dial Transplant 2001; 16: 1542-5.

7. Santoro A. Confounding factors in the assessment of delivered hemodialisys dose. Kidney Int 2000; 58 (Suppl. 76): S19-27.

8. Cimino JE, Brescia MJ. Simple venipuncture for hemodialysis. NEngl J Med 1962; 267: 608.
9. Hirth RA, Turenne MN, Woods JD, et al. Predictors of type of vascular access in hemodialysis patients. JAMA 1996; 276: 1303-8.

10. Baker LD, Johnson JM, Goldfarb D. Expanded polytetrafluoroethylene (PTFE) subcutaneous arterio-venous conduit: an improved vascular access for chronic hemodialysis. Trans Am Soc Artif Intern Organs 1976; 22: 3827.

11. Canaud B. Haemodialysis catheter-related infection: time for action. Nephrol Dial Transplant 1999; 14: 2288 90 .

12. Ha ancora senso fare emodialisi domiciliare nel 2001? A cura di Marco Lombardi. Giornale di Tecniche Nefrologich \& Dialitiche 2001; 13 (Suppl. 2): S3-74.

13. Stehman-Breen CO, Sherrard DJ, Gillen D, Caps M. Determinants of type and timing of initial permanent hemodialy sis vascular access. Kidney Int 2000; 57: 639-45.

14. Van Biesen W, Vanholder R, Lameire N, The role of peritoneal dialysis as the first line renal replacement modality. Perit Dial Int 2000; 20: 375-83.

15. http://www.sin-italia.org/gruppi/ vascolare/ Gruppo di Studio per gli Accessi Vascolari. 